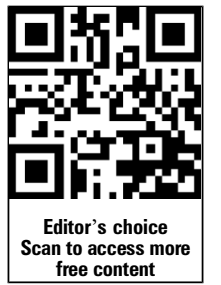

- Additional material is published online only. To view please visit the journal online (http://dx.doi.org/10.1136/ heartjnl-2013-303722).

\section{${ }^{1}$ Multidisciplinary} Cardiovascular Research Centre, Leeds Institute of Genetics, Health and Therapeutics, University of Leeds, Leeds, UK

${ }^{2}$ Academic Unit of Health Economics, Leeds Institute of Health Sciences, University of Leeds, Leeds, UK ${ }^{3}$ Department of Cardiology, Leeds General Infirmary, Leeds, UK

\section{Correspondence to} Dr J P Greenwood, Multidisciplinary Cardiovascular Research Centre, Leeds Institute of Genetics, Health and Therapeutics, University of Leeds, Leeds LS1 3EX, UK; j.greenwood@leeds.ac.uk

Received 24 January 2013 Revised 26 March 2013 Accepted 27 March 2013 Published Online First 21 May 2013
To cite: Fairbairn TA, Meads DM, Hulme C, et al. Heart 2013;99:914-920.

\title{
The cost-effectiveness of transcatheter aortic valve implantation versus surgical aortic valve replacement in patients with severe aortic stenosis at high operative risk
}

\author{
Timothy A Fairbairn, ${ }^{1}$ David M Meads, ${ }^{2}$ Claire Hulme, ${ }^{2}$ Adam N Mather, ${ }^{1}$ Sven Plein, ${ }^{1}$ \\ Daniel J Blackman, ${ }^{3}$ John P Greenwood ${ }^{1}$
}

\begin{abstract}
Objective To determine the cost-effectiveness of transcatheter aortic valve implantation (TAVI) compared with surgical aortic valve replacement (SAVR) in a highrisk aortic stenosis (AS) population.
\end{abstract}

Design A cost-utility analysis employing the National Institute of Clinical Excellence (NICE) reference case design for technology appraisals.

Setting The perspective of the UK National Health

Service.

Patients Utility data from a UK high-risk AS population. TAVI and SAVR effectiveness was taken from the PARTNER A randomised controlled trial.

Main outcome measures Costs modelled over a 10 year horizon using a Markov model. Incremental costeffectiveness ratios and cost-effectiveness acceptability curve were calculated with reference to the NICE willingness to pay per quality adjusted life year (QALY) gain threshold. Deterministic and probabilistic sensitivity analyses performed.

Results Despite greater procedural costs ( $£ 16500$ vs $\mathrm{f9}, 256)$, TAVI was cost-effective compared with SAVR over the 10 year model horizon (costs $£ 52593$ vs f53 943 and QALYs 2.81 vs 2.75), indicating that TAVI dominated SAVR. This appeared to be due to greater postsurgical costs, related to the length and cost of hospital stay. The results appeared robust to a number of deterministic sensitivity and probabilistic analyses. The cost-effectiveness acceptability curve indicated that at the NICE $f 20000$ willingness to pay threshold per QALY gained, TAVI had a $64.6 \%$ likelihood of being costeffective, compared with $35.4 \%$ for SAVR.

Conclusions TAVI is likely to be a cost-effective treatment for high-risk patients with AS compared with the reference standard of SAVR. However, uncertainty surrounding the long-term outcomes for TAVI patients remains; this could have a substantive impact on estimates of cost-effectiveness.

\section{INTRODUCTION}

Aortic stenosis (AS) is the most common valvular heart disease in the western world. As a predominantly degenerative process the disease prevalence increases with age, affecting $4 \%$ of individuals aged 85 years and older. ${ }^{1}{ }^{2}$ The onset of symptoms predict a poor prognostic outlook and a reduced quality of life, with valve replacement the only successful treatment option. ${ }^{3}{ }^{4}$ The European Heart
Survey demonstrated that a third of suitable patients did not receive definitive surgical treatment due to factors such as age, left ventricular dysfunction and associated comorbidities. ${ }^{5}$ Transcatheter aortic valve implantation (TAVI) developed as an alternative procedure for those individuals deemed at high risk or inoperable for conventional surgical aortic valve replacement (SAVR). TAVI improves survival, functional capacity and quality of life compared with standard medical therapy ${ }^{6}$ and at 2 years is non-inferior to SAVR. ${ }^{7}{ }^{8}$ However there remains a question regarding the cost-effectiveness of this intervention.

The aim of this study was to determine whether TAVI is a cost-effective alternative to SAVR in a high-risk group by developing a decision-analytic economic model using the available published evidence and values that reflect UK costs and clinical practice. We aim to provide an estimate of the average cost-effectiveness of TAVI across access site (transfemoral (TF) or transapical (TA)) and valve brands (Edwards-SAPIEN and Medtronic CoreValve) with sensitivity analyses to cover differential performance according to these factors; this is to ensure model results reflect an overall viewpoint for the patient, clinician and purchaser in a healthcare system, where differential practice may occur.

\section{METHODS}

Health costs and outcomes relating to TAVI and SAVR were assessed by combining health-related quality of life data from a UK AS population with data extracted from published randomised and registry studies.

\section{Analyses and model structure}

A cost-utility analysis was conducted with benefits expressed in terms of quality adjusted life years (QALYs), ${ }^{9}$ costs presented from the perspective of the UK healthcare provider and results expressed as incremental cost-effectiveness ratio (ICERs). ${ }^{10}$ A decision tree was constructed to capture the costs and benefits of the interventions from baseline to 2 years and a cohort Markov Model with annual cycles was used to propagate the costs and benefits over a 10 year time horizon (see online supplementary appendix A). A supplementary analysis was based on life years provided by the interventions. 
Costs and benefits after year 1 were discounted at the National Institute of Clinical Excellence (NICE) preferred rate of 3.5\%. Analyses were conducted in Microsoft Excel (Microsoft Corporation, Redmond, Washington, USA).

\section{Outcomes and utility}

Health benefits were based on New York Heart Association (NYHA) class transitions with each class ascribed a mean EQ-5D (EuroQoL, 1990) health utility value generated from a UK study population previously described. ${ }^{11}$ This population data did not include any NYHA category I patients, therefore EQ-5D values for this group were taken from UK population norms for the age group. ${ }^{12}$ As the only randomised study of TAVI versus SAVR in high-risk patients, PARTNER A was used as the basis of the patient outcomes data. ${ }^{8}$ The NYHA proportions from the trial publication were employed with the local NYHA mean EQ-5D values, to generate baseline to 2 year QALYs. As NYHA transition may not capture all the impact of complications additional utility decrements were calculated by subtracting mean utility values associated with complications in published literature from the mean EQ-5D scores for NYHA III. These were adjusted to $80 \%$ to reflect the proportion of patients with complications that would die. The NYHA proportions at 2 years were subject to the proportional changes observed in NYHA classes by Kodali et al from 1 year to 2 years. The changes from 1 year to 2 years were employed as constant proportional changes (with half-cycle correction) in NYHA classes for the 10 year time horizon of the cohort (table 1). To illustrate, the population of NYHA category I was reduced by $12 \%$ annually. Using this approach and considering the percentage reductions from the other NYHA categories, the annual mortality rate was set at $40 \%$.

\section{Costs}

Costs were based on national UK values (table 2). The TAVI procedure was charged at a standard NHS tariff payment-by-results fee. This fee covered the device costs, procedural costs (medical professionals, theatre time), postoperative recovery (coronary care unit) and 4 days general ward hospital stay. The TAVI care pathway incorporated 4 additional days general ward hospital stay, ambulatory monitoring, two echocardiograms, ECGs, a vascular surgery consultation, and three follow-up visits at 1 month, 6 months and 12 months. The SAVR clinical pathway was similar except that this group had 5 days in an intensive therapy unit bed, 7 days in a general ward bed. Long-term costs up to 2 years included cost of the procedure, valve redos, length of hospital stay, complications and medication requirements. Future costs were calculated per NYHA category based on
Table 2 Costs of the standard procedure, postprocedural complications, long-term medications and healthcare

\begin{tabular}{|c|c|c|}
\hline Unit Cost (source) & TAVI & SAVR \\
\hline \multicolumn{3}{|l|}{ Standard Procedure (NHS tariff) } \\
\hline Procedure & $£ 16500.00$ & £9256.00 \\
\hline Cardiology-ambulatory monitoring & f25.65 & f25.65 \\
\hline $\begin{array}{l}\text { Vascular surgery—follow-up attendance—single } \\
\text { professional }\end{array}$ & f120.00 & NA \\
\hline CT Angioaortic and peripheral & f148.14 & NA \\
\hline Transthoracic echo (ultrasound) & f27.64 & $\mathrm{f} 27.64$ \\
\hline Cardiology_ultrasound transoesophageal echo & f128.24 & f128.24 \\
\hline Cardiology—ECG & f33.00 & £33.00 \\
\hline Chest physiotherapy & f1641.00 & £1641.00 \\
\hline Cardiology follow-up attendance & f113.00 & $£ 113.00$ \\
\hline General ward bed day cost & $f 280.00$ & f280.00 \\
\hline Intensive care unit bed day cost & f1360.00 & f1360.00 \\
\hline \multicolumn{3}{|l|}{ Complication unit costs (NHS Ref costs) } \\
\hline Pacemaker implant & $£ 2886.00$ & £2886.00 \\
\hline TIA & f1252.00 & £1252.00 \\
\hline Minor stroke & f3479.00 & f3479.00 \\
\hline Major stroke & f3479.00 & f3479.00 \\
\hline Myocardial infarct & $£ 2305.00$ & $£ 2305.00$ \\
\hline Vascular complication & f3772.34 & f3772.34 \\
\hline Major bleed & £3772.00 & $£ 3772.00$ \\
\hline RR for kidney fail & f1421.00 & f1421.00 \\
\hline Endocarditis & f5261.00 & f5261.00 \\
\hline Repeat hospitalisation & f1359.00* & £1359.00* \\
\hline \multicolumn{3}{|l|}{ Cost of care per NYHA category (PSSRU) $\beta$} \\
\hline Dead & f0.00 & $f 0.00$ \\
\hline 1 & f55.00 & $£ 55.00$ \\
\hline II & f141.00 & f141.00 \\
\hline III & f223.00 & f223.00 \\
\hline IV & f626.00 & f626.00 \\
\hline \multicolumn{3}{|l|}{ Annual medication costs (BNF) $\beta$} \\
\hline Clopidogrel for stroke, TIA and MI & f30.00 & f30.00 \\
\hline Secondary care anticoagulation services for AF & f649.00 & f649.00 \\
\hline$\beta$ blockers for $\mathrm{Ml}$ & f19.66 & f19.66 \\
\hline Simvastatin & f12.09 & f12.09 \\
\hline ACE inhibitors for MI & f14.75 & f14.75 \\
\hline
\end{tabular}

weekly care package and subsequent hospitalisation tariffs calculated using a previously published hospitalisation annual hazard per NYHA category. ${ }^{13}$

Table 1 Utility scores and NYHA proportional changes

\begin{tabular}{|c|c|c|c|c|c|c|c|}
\hline \multicolumn{3}{|c|}{ EQ5D utility scores } & \multicolumn{2}{|c|}{$\begin{array}{l}\text { Annual NYHA Changes from } \\
2 \text { years }\end{array}$} & \multicolumn{3}{|l|}{ Utility decrements } \\
\hline NYHA & & Reference & $\%$ change & Reference & Complication & Decrement & Reference \\
\hline I & 0.73 & UK Norm, Kind et al ${ }^{12}$ & -0.12 & Kodali et al $\left.\right|^{8}$ & Major stroke & 0.39 & Solomon et $a l^{31}$ \\
\hline ॥ & 0.63 & Fairbairn et $\left.a\right|^{11}$ & -0.14 & Kodali et $a l^{8}$ & Vascular complication & 0.06 & Morgan et $a l^{32}$ \\
\hline III & 0.56 & Fairbairn et $a l^{11}$ (2012) & -0.06 & Kodali et $a l^{8}$ & Renal replacement therapy & 0.11 & Lee et $a l^{\beta 3}$ \\
\hline IV & 0.46 & Fairbairn et $a l^{11}$ (2012) & -0.08 & Kodali et $a l^{8}$ & & & \\
\hline Dead & 0 & $\mathrm{~N} / \mathrm{A}$ & 40 & Kodali et $a l^{8}$ & & & \\
\hline
\end{tabular}

NYHA, New York Heart Association. 
Table 3 Event probabilities and their reference source

\begin{tabular}{|c|c|c|c|c|}
\hline & \multicolumn{2}{|l|}{ TAVI } & \multicolumn{2}{|l|}{ SAVR } \\
\hline & Probability & Reference & Probability & Reference \\
\hline \multicolumn{5}{|l|}{ Procedural outcomes } \\
\hline Conversion to SAVR/TAVI & 0.007 & 30 & 0.003 & 7 \\
\hline Multiple valve ( $\geq 2$ implanted) & 0.02 & 7 & 0 & $\mathrm{~N} / \mathrm{A}$ \\
\hline Intensive care unit bed days (n) & 0.5 & $\begin{array}{l}\text { LTHT } \\
\text { Expert opinion }\end{array}$ & 5 & 7,21 \\
\hline Coronary care bed days (n) & 3 & $\begin{array}{l}\text { LTHT } \\
\text { Expert opinion }\end{array}$ & 0 & $\begin{array}{l}\text { LTHT } \\
\text { Expert opinion }\end{array}$ \\
\hline General ward bed days ( $n$ ) & 4 & $\begin{array}{l}\text { LTHT } \\
\text { Expert opinion }\end{array}$ & 7 & $\begin{array}{l}21 \\
\text { LTHT } \\
\text { Expert opinion/ }\end{array}$ \\
\hline \multicolumn{5}{|l|}{2 Year complications } \\
\hline New permanent pacemaker & 0.15 & Mean MCV/ESV & 0.05 & 7 \\
\hline TIA & 0.036 & 8 & 0.020 & 8 \\
\hline Stroke & 0.077 & 8 & 0.049 & 8 \\
\hline Myocardial infarction & 0.004 & 8 & 0.015 & 8 \\
\hline Major vascular complication & 0.116 & 8 & 0.038 & 8 \\
\hline Major bleed & 0.19 & 8 & 0.295 & 8 \\
\hline RR for kidney failure & 0.062 & 8 & 0.069 & 8 \\
\hline Endocarditis & 0.015 & 8 & 0.01 & 8 \\
\hline New atrial fibrillation & 0.12 & 7 & 0.17 & 7 \\
\hline \multicolumn{5}{|l|}{ Hospitalisation hazard by NYHA } \\
\hline 1 & 0.26 & 13 & & \\
\hline$\|$ & 0.42 & 13 & & \\
\hline III & 0.79 & 13 & & \\
\hline IV & 1.81 & 13 & & \\
\hline Dead & 0 & $\mathrm{~N} / \mathrm{A}$ & & \\
\hline
\end{tabular}

\section{Event probabilities}

The majority of event probabilities were taken from the PARTNER A study (table 3). Due to variations in pacemaker implantation post-TAVI according to the valve type an average of probabilities was taken from previous studies to reflect mean event rates.

Long-term data relating to the outcomes of patients with TAVI or the longevity of the valve are not yet available, hence a number of assumptions were necessary: (1) The TAVI valve retains functionality for the lifetime of the patient. (2) Implanted pacemakers do not require replacement. (3) Patients with TAVI and SAVR are subject to the same NYHA proportional change rate after 2 years. In addition, it was also assumed that utility decrements associated with complications were experienced for the first 2 years only, as patients with serious complications are more likely to die early in the model.

\section{Sensitivity analyses}

Deterministic and probabilistic sensitivity analyses (PSA) were conducted to evaluate the sensitivity of the results to the assumptions made and parameter values chosen ${ }^{14}$ and to determine the level of uncertainty surrounding the base case estimates. Since the increased likelihood of early major stroke is a concern with the TAVI procedure, additional sensitivity analyses were run to explore the impact of this complication on the costeffectiveness results.

For the PSA, distributions for parameter values were specified and 10000 Monte Carlo simulations run using random draws for each parameter distribution (see online supplementary appendix B) and for each run incremental costs and benefits calculated. The outcomes of the PARTNER A trial (the NYHA transitions from baseline to 2 years) were assumed to be fixed. The PSA allowed the NYHA lifetime changes to vary independently for TAVI and SAVR. The uncertainty surrounding the analyses were represented as incremental benefit and cost plots for each simulation run on a cost-effectiveness plane. Net benefit was calculated and a cost-effectiveness acceptability curve $(\mathrm{CEAC})^{15}$ was generated to determine the probability that TAVI was cost-effective versus SAVR given a range of values of willingness to pay for an additional QALY.

\section{RESULTS}

\section{Base case analysis}

The NYHA proportions and mortality for the two groups over the 10-year horizon are shown (see online supplementary appendix C). As the NYHA proportional changes were assumed the same across TAVI and SAVR the differential in costs and effects remains relatively constant after year 1 and across the model time horizon. TAVI ranges between $£ 1,350$ and $£ 1,600$ per patient cheaper than SAVR annually. After year 1 the annual QALY differences between interventions are negligible (around 0.003-0.005 in favour of TAVI). After year 3 there was little difference between interventions in terms of mortality. By year 10 in the simulations, $75 \%$ and $76 \%$ of the cohort were dead in the TAVI and SAVR groups, respectively. At 2 years TAVI was found to confer QALY gains of 0.956 compared with 0.925 provided by SAVR. After 10 years the average QALY gains per person were 2.81 and 2.75 , respectively, providing a modest 
Figure 1 (A) Hospital admission costs of transcatheter aortic valve implantation (TAVI) and surgical aortic valve replacement (SAVR)

(B) Cost-effectiveness plane

(C) Cost-effectiveness acceptability curve. This figure is only reproduced in colour in the online version.

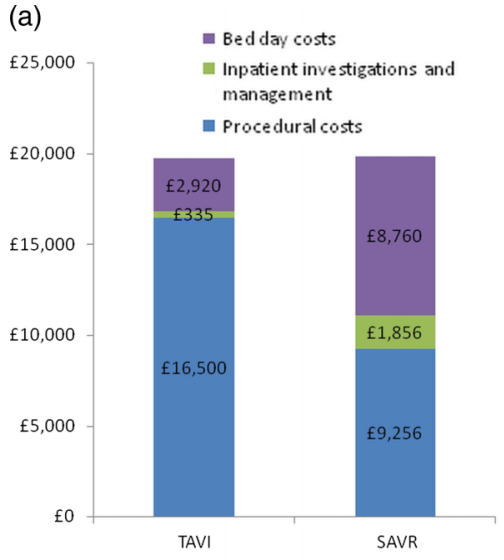

(b)

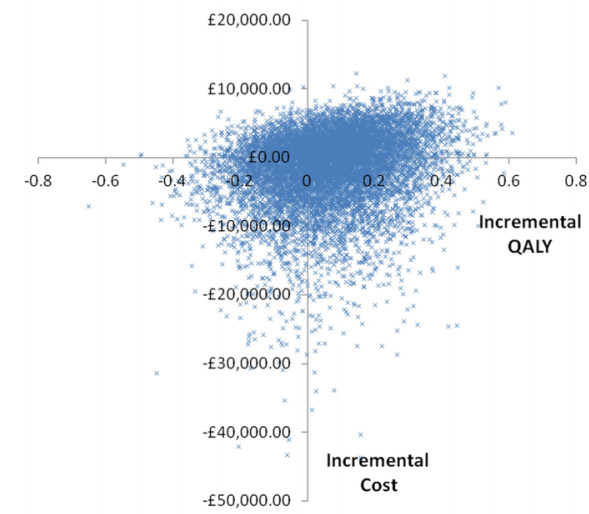

(c)

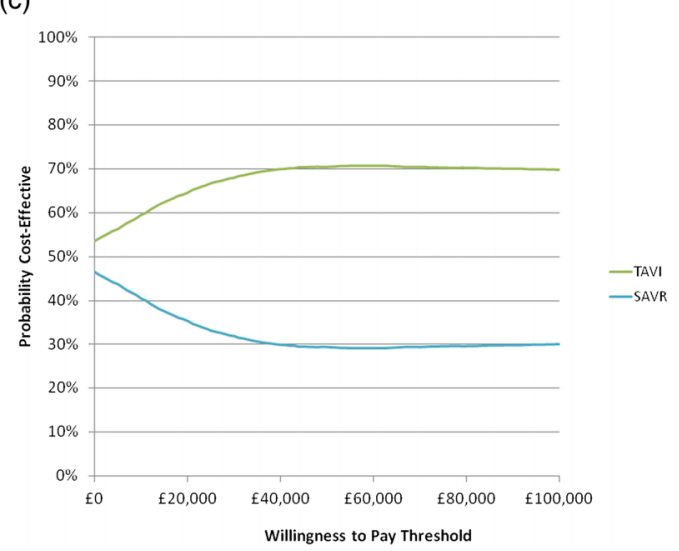

incremental benefit for TAVI of 0.063 QALYs. Procedural costs were estimated to be $£ 19368$ for TAVI and $£ 20380$ for SAVR. While the TAVI tariff was substantially more than the SAVR tariff, the surgical intervention incurred greater length of stay and time in intensive care which are significant drivers of cost (figure 1A). Complication costs were similar across the interventions though slightly higher in SAVR presumably due to the high costs associated with endocarditis and new atrial fibrillation (both more likely in SAVR). The long-term care and hospitalisation costs over the 10 year time horizon were similar in TAVI and SAVR which is unsurprising as they were based on the NYHA proportions. The slightly lower long-term costs in SAVR may be due to the higher mortality in this group. Total 10 year per person costs, benefits (QALYs and life years) and the respective ICER are included in table 4. The base case analysis figures indicate that TAVI dominates SAVR - that is, it is cheaper and more effective-over the time horizon.

\section{Deterministic sensitivity and scenario analyses}

Deterministic sensitivity analysis and PSA are presented in table 4. These suggest the base case results are robust to changes in input parameters, yielding similar ICERs. Only analyses where parameter values for one intervention changed (and the other held the same) appeared to have a substantive effect on the ICER value. Thus using the worst case scenario 1 year complication probabilities for TAVI yielded an ICER of $£ 11307$-in this case TAVI is now more expensive but still offers incremental benefit. Increasing the TAVI procedural costs by $25 \%$ increased the ICER to just over $£ 54000$. Allowing for 2 bed days in intensive care ${ }^{16}$ for patients with TAVI increases the ICER to around $£ 11000$ - still below the NICE cost-effectiveness threshold. Including 3 days of intensive care for TAVI yields an ICER of $£ 32660$. The cost of the SAVR tariff would have to drop to $£ 6632$ (ceteris paribus) or alternatively incur only around 3 intensive care bed days before the ICER exceeds the $£ 20000$ threshold. TAVI tariff costs would have to be around $£ 19000$ or $£ 9,800$ more expensive than the SAVR tariff, for the ICER to exceed $£ 20000$. The alternative utility values did not significantly affect results, yielding slightly higher incremental benefits $(0.066$ vs 0.063$)$ for TAVI. Reducing the time horizon to 5 years and alternating discount rates did not substantively affect results.

TAVI remained dominant in the instance of increasing the probability of major stroke after TAVI (from 0.051 to 0.10 ) or increasing the utility decrement associated with stroke (from 0.39 to 0.70 ). Finally, if all values remain constant as per the base case, the cost of stroke would have to be increased to over $£ 100000$ (from $£ 3,479$ ) before the ICER exceeded $£ 20000$.

\section{Probabilistic sensitivity analyses}

Figure $1 \mathrm{~B}$ is the cost-effectiveness plane plotting 10000 incremental cost and benefit estimates from the Monte Carlo simulations. Most of the estimates are in the North-East and South-East quadrants indicating TAVI is more costly and more effective or cheaper and more effective. Figure $1 \mathrm{C}$ is the CEAC, indicating TAVI is cost-effective regardless of the incremental QALY willingness to pay threshold. At the NICE threshold of $£ 20000$ TAVI has a $64.6 \%$ likelihood of being cost-effective, compared with $35.4 \%$ for SAVR. The mean incremental costs and benefits from the Monte Carlo simulations (table 4) were very similar to the base case estimate.

\section{DISCUSSION}

The economic analysis in this study suggests that from the UK healthcare provider perspective, TAVI is a cost-effective option 
Table 410 year deterministic and sensitivity analyses results

\begin{tabular}{|c|c|c|c|}
\hline & Costs* & QALYst & ICER \\
\hline \multicolumn{4}{|l|}{ Base case } \\
\hline TAVI & & 2.81 & \\
\hline Procedural and redo costs & £19368.32 & & \\
\hline Complication costs (after 2 years) & £2125.14 & & \\
\hline Annual medication costs (after 2 years) & f82.63 & & \\
\hline Long-term care and rehospitalisation costs & f31422.01 & & \\
\hline TAVI total 10 year cost & f52593.02 & & \\
\hline SAVR & & 2.75 & \\
\hline Procedural and redo costs & f20380.03 & & \\
\hline Complication costs & $£ 2709.60$ & & \\
\hline Annual medication costs & $£ 113.62$ & & \\
\hline Long-term care and rehospitalisation costs & £31095.10 & & \\
\hline SAVR total 10 year costs & f53943.40 & & \\
\hline Incremental QALY & $-\mathrm{f} 1350.38$ & 0.063 & TAVI dominates \\
\hline Life years & 4.42 & 4.30 & \\
\hline Incremental life years & $-f 1350.38$ & 0.13 & TAVI dominates \\
\hline Deterministic sensitivity analyses & Incremental cost & Incremental QALY & ICER \\
\hline \multicolumn{4}{|l|}{ Costs } \\
\hline Procedural costs $+25 \%$ & $-f 1689.63$ & 0.063 & TAVI dominates \\
\hline Procedural costs $-25 \%$ & $-\mathrm{f} 1011.13$ & 0.063 & TAVI dominates \\
\hline TAVI tariff price $f 25,000$ & f7294.12 & 0.063 & f116231.63 \\
\hline TAVI procedure costs $+25 \%$ & f3393.00 & 0.063 & f54067.41 \\
\hline TAVI length of stay alternative values & f689.62 & 0.063 & £10989.06 \\
\hline Complication costs $+25 \%$ & $-\mathrm{f} 1497.51$ & 0.063 & TAVI dominates \\
\hline Complication costs $-25 \%$ & $-f 1203.25$ & 0.063 & TAVI dominates \\
\hline Hospitalisation costs $+25 \%$ & $-\mathrm{f} 1336.36$ & 0.063 & TAVI dominates \\
\hline Hospitalisation costs $-25 \%$ & $-\mathrm{f} 1364.40$ & 0.063 & TAVI dominates \\
\hline Weekly care costs by NYHA $+25 \%$ & $-\mathrm{f} 1264.95$ & 0.063 & TAVI dominates \\
\hline Weekly care costs by NYHA $-25 \%$ & $-\mathrm{f} 1435.81$ & 0.063 & TAVI dominates \\
\hline \multicolumn{4}{|l|}{ Utility } \\
\hline Alternative utility values & $-\mathrm{f} 1350.38$ & 0.066 & TAVI dominates \\
\hline Complication utility decrement $+25 \%$ & $-\mathrm{f} 1350.38$ & 0.058 & TAVI dominates \\
\hline \multicolumn{4}{|l|}{ Event probabilities } \\
\hline TAVI extreme ('worst case') scenario complication Probabilities & f99.95 & 0.009 & £11,307.18 \\
\hline UK Registry TAVI complication probabilities & $-\mathrm{f} 1715.18$ & 0.076 & TAVI dominates \\
\hline Hospitalisation rates by NYHA $+25 \%$ & $-\mathrm{f} 1336.36$ & 0.063 & TAVI dominates \\
\hline Hospitalisation rates by NYHA $-25 \%$ & $-\mathrm{f} 1364.40$ & 0.063 & TAVI dominates \\
\hline \multicolumn{4}{|l|}{ Other } \\
\hline Time horizon $=5$ years & $-\mathrm{f} 1452.91$ & 0.045 & TAVI dominates \\
\hline Discount rate $1 \%$ for costs and QALYs & $-\mathrm{f} 1323.06$ & 0.067 & TAVI dominates \\
\hline Discount rate $6 \%$ for costs and QALYs & $-£ 1373.57$ & 0.059 & TAVI dominates \\
\hline \multicolumn{4}{|l|}{ Probabilistic sensitivity analysis - mean Monte Carlo simulation results } \\
\hline TAVI & £52593.08 & 2.82 & \\
\hline SAVR & f54004.89 & 2.75 & \\
\hline Incremental & $-f 1411.09$ & 0.066 & TAVI dominates \\
\hline
\end{tabular}

in high-risk but operable elderly patients when compared with SAVR. Over a 10 year horizon, the model yielded incremental cost and QALY benefits for TAVI over SAVR. These results appear robust to numerous sensitivity analyses including those targeting major stroke. TAVI conferred only marginal quality of life benefits over SAVR with similar costs for both interventions. Therefore, results were sensitive to changes in costs and benefits when they occurred in either intervention arm in isolation. The additional device costs for TAVI appears outweighed by the greater length of stay cost (and intensive care stay) in the SAVR group. The PSA and CEAC suggest that TAVI is probably cost-effective regardless of the QALY willingness to pay threshold.

The cost-effectiveness of TAVI versus medical therapy has been previously assessed in a UK population ineligible for surgery, ${ }^{17}$ but this analysis is the first UK study to compare the 
costs and benefits of TAVI and SAVR in a high-risk but operable AS group. The recently published PARTNER study costeffectiveness analysis reports a significant cost benefit at 1 year towards TF-TAVI with similarly higher procedural but lower hospitalisation costs compared with SAVR. ${ }^{18}$ Procedural costs are likely to differ between studies (US versus UK costs) but health benefits (QALYs) should remain universal. The 1 year incremental QALYs in both analyses are 0.027 supporting our model design, but this study reports lower QALY gains with TAVI. This may be a consequence of our combined TF and TA assessment as TA-TAVI resulted in lower quality adjusted life expectancy and was deemed economically unattractive in the PARTNER study. Another cost-analysis by Neyt et al ${ }^{19}$ concluded that TAVI was cost-effective in inoperable patients but not in high-risk operable patients. However, their conclusions are limited by a lack of health utility data. Doble et $a l^{20}$ performed a comprehensive analysis of TAVI versus medical and surgical therapy concluding that TAVI may not be cost-effective compared with SAVR over a 20 year horizon. This observation was determined predominantly by the higher procedural costs of TAVI. However, their SAVR costs were estimated using a lower-risk, 70 year-old population rather than the older, higherrisk population in this study and that of PARTNER. In addition there is no mention of the length or cost of hospital stay, a major driver in our own cost analysis. This study has shown that despite a greater procedural cost, TAVI remained cost-effective compared with SAVR due to lower postprocedural costs (length and cost of hospital stay). Our TAVI group had a mean Intensive care unit (ICU) stay of 0.5 days and 3 further days in a coronary care unit. In the PARTNER A study there was a median ICU stay of 3 days reflective of US practice. If outcomes are in part a function of the intensity of care received, it is possible we overestimated outcomes or underestimated the costs for TAVI depending upon individual practice and national guidance. However, including 2.4 days of intensive care for patients with TAVI in this analysis still yields an ICER below $£ 20000$. Additionally, the ICU stay post-SAVR in PARTNER A may be considered longer than is 'normal' for a standard SAVR postoperative recovery. The nature of high-risk cardiothoracic surgery in this older age group with a number of associated comorbidities results in a longer ICU and overall hospital stay, thus driving up costs. ${ }^{21}$ This study used the UK TAVI tariff as an average national procedural cost with sensitivity analysis to allow for variations which may occur locally. Transcatheter device costs are of particular concern as a driver of high procedural costs. Our results suggest that TAVI is no longer costeffective when device costs rise above $£ 19000$ (when the QALY gain willingness to pay threshold is $£ 20000$ ).

Post-TAVI we assumed no valve redos after 12 months and that the integrity of valves was maintained for the model time horizon. Thus our results would be sensitive to any future evidence suggesting differential rates of valve failure and redo procedures between SAVR and TAVI. Due to the uncertainties of long-term data, our model time horizon was performed at a conservative 10 years rather than a lifetime model. In their lifetime comparison of TAVI versus standard medical therapy, Reynolds et $a l^{22}$ found significant cost benefits of TAVI within the 1st year, which were subsequently lost over long-term follow-up due to the burden of incurred costs over an extended lifetime in an elderly population. This issue may not apply in a younger population with less comorbidity, as studies have demonstrated that most long-term deaths post-TAVI are noncardiac in origin. ${ }^{23-25}$ However, the shorter timeframe of the model and uncertainty in terms of outcomes, device longevity and need for future valve replacements post-TAVI, mean that extrapolation of our findings to a younger, lower-risk age group remains difficult. Stroke is a significant clinical concern post-TAVI. $^{7}$ As a major contributor to hospital and social care costs, ${ }^{26}$ stroke could additionally impact upon the costeffectiveness of the TAVI procedure. However, our analysis suggests that TAVI still dominates SAVR in cost-effectiveness even in the presence of a doubling of the stroke rate. This finding is reassuring, particularly when considering the latest evidence from PARTNER which suggests that the 2 year stroke rate is not significantly different between TAVI and SAVR.

Our findings offer a perspective based on UK clinical practice, costs and local health utility values. The results provide useful information to local healthcare commissioning agencies and national policy makers regarding the relative cost-effectiveness of TAVI and SAVR in elderly high-risk patients. The clinical effectiveness of TAVI has been demonstrated in the PARTNER A trial; our study provides additional evidence that compared with SAVR, TAVI is likely to be a cost-effective approach in an elderly high-risk AS population.

There is a clear requirement for longer term outcome data in patients with TAVI. Such data will become available as the TAVI procedure becomes more common in clinical practice and large data registries are published. Avenues for future research in health economics include the employment of the value of information framework and the calculation of total decision uncertainty (expected value of perfect information) ${ }^{27}$ for parameters and samples. This information will help identify the parameters that are driving economic decision uncertainty and guide future research and trial planning.

\section{Limitations}

As TAVI is a relatively new procedure there are limited data available to populate the decision model. Hence a number of assumptions were necessary which increase the level of uncertainty in the results. However, sensitivity analyses suggest our results are relatively unaffected by changes in parameter values.

While we have based costs on the UK care pathway for this population we have based the benefits on US data (PARTNER A). The PARTNER A trial employed TA and TF implantation approaches but only the Edward Sapien device was used. Reliance on the PARTNER A study efficacy data may limit extrapolation of our findings outside of the studies recruitment criteria, in particular related to the Medtronic CoreValve system. In addition the PARTNER A cost-effectiveness study reports a 0.068 QALY gain with TF-TAVI, but 0.070 loss with TA-TAVI. This suggests a potential weakness in our methodology of reporting an average costeffectiveness despite the use of deterministic sensitivity analysis and PSA. Future research should explore the differential costeffectiveness of TA and TF approaches in the UK.

\section{CONCLUSIONS}

With any new treatment, the medical community must ask first if it is safe and clinically effective and second if it is cost-effective. In comparison with the accepted reference standard treatment SAVR, TAVI appears likely to be cost-effective in a high-risk elderly population. TAVI was cheaper and more effective than SAVR according to the base case analysis. Sensitivity analysis using the NICE threshold of $£ 20000$ showed TAVI to have a 64.6.1\% likelihood of being cost-effective, compared with $35.4 \%$ for SAVR. While the findings cannot be extended to other populations of different age or surgical risk, the evidence provided should help clinicians and commissioning groups in future decision-making policies and resource allocation. 
Contributors All authors fulfil the International Committee of Medical Journal Editors (ICMJE) uniform requirements for manuscript submission. JPG as corresponding author acts as guarantor. TAF was involved in the study conception and design; acquisition, analysis and interpretation of data; drafted the article and had final approval of the manuscript. DMM was involved in the study design, model construction and interpretation of results; drafted the article and had final approval of the manuscript. $\mathrm{CH}$ was involved in the study design and interpretation of data; revising the article and had final approval of the manuscript. ANM was involved in the acquisition and analysis of data; revising the article and had final approval of the manuscript. SP was involved in the study design and interpretation of data; revising the article and had final approval of the manuscript. DJB was involved in the study design and interpretation of data; revising the article and had final approval of the manuscript. JPG was involved in the study conception and design; interpretation of data; drafting and revising the article and had final approval of the manuscript.

Funding The work was funded by the British Heart Foundation (PG/11/126/29321). Competing interests None.

Ethics approval Leeds (West) Research Ethics Committee (08/H1307/106).

Provenance and peer review Not commissioned; externally peer reviewed.

Data sharing statement All authors, external and internal, had full access to all of the data (including statistical reports and tables) in the study and can take responsibility for the integrity of the data and the accuracy of the data analysis. No additional unpublished data are available.

\section{REFERENCES}

1 Nkomo VT, Gardin JM, Skelton TN, et al. Burden of valvular heart diseases: a population-based study. Lancet 2006;368:1005-11.

2 Carabello BA, Paulus WJ. Aortic stenosis. Lancet 2009:373:956-66.

3 Pellikka PA. Outcome of 622 Adults With Asymptomatic, Hemodynamically Significant Aortic Stenosis During Prolonged Follow-Up. Circulation 2005;111:3290-5.

4 Kvidal P, Bergstrom R, Horte LG, et al. Observed and relative survival after aortic valve replacement. J Am Coll Cardiol 2000:35:747-56.

5 lung B, Cachier A, Baron G, et al. Decision-making in elderly patients with severe aortic stenosis: why are so many denied surgery? Eur Heart J 2005:26:2714-20.

6 Leon MB, Smith CR, Mack M, et al. Transcatheter aortic-valve implantation for aortic stenosis in patients who cannot undergo surgery. N Engl J Med 2010:363:1597-607

7 Smith CR, Leon MB, Mack MJ, et al. Transcatheter versus surgical aortic-valve replacement in high-risk patients. N Engl J Med 2011;364:2187-98.

8 Kodali SK, Williams MR, Smith CR, et al. Two-year outcomes after transcatheter or surgical aortic-valve replacement. N Engl J Med 2012;366:1686-95.

9 Richardson G, Manca A. Calculation of quality adjusted life years in the published literature: a review of methodology and transparency. Health Econ 2004; 13:1203-10.

10 Reed SD, Anstrom KJ, Bakhai A, et al. Conducting economic evaluations alongside multinational clinical trials: toward a research consensus. Am Heart J 2005; 149:434-43.

11 Fairbairn TA, Meads DM, Mather AN, et al. Serial change in health-related quality of life over 1 year after transcatheter aortic valve implantation: predictors of health outcomes. J Am Coll Cardiol 2012;59:1672-80.

12 Kind P HG, Macran S. UK Population norms for EQ-5D. York Centre for Health Economics. 1999 1999;University of York.

13 Caro JJ, Guo S, Ward A, et al. Modelling the economic and health consequences of cardiac resynchronization therapy in the UK. Curr Med Res Opin 2006;22:1171-9.
14 Maliwa MA, van der Heijden GJ, Bots ML, et al. Quality of life and NYHA class 30 years after mechanical aortic valve replacement. Cardiovasc Surg 2003;11:381-7.

15 Fenwick E, Claxton K, Sculpher M. Representing uncertainty: the role of cost-effectiveness acceptability curves. Health Econ 2001;10:779-87.

16 Zahn R, Gerckens U, Grube E, et al. Transcatheter aortic valve implantation: first results from a multi-centre real-world registry. European Heart Journal 2010;32:198-204.

17 Watt M, Mealing S, Eaton J, et al. Cost-effectiveness of transcatheter aortic valve replacement in patients ineligible for conventional aortic valve replacement. Heart 2012:98:370-6

18 Reynolds MR, Magnuson EA, Lei Y, et al. Cost-Effectiveness of transcatheter aortic valve replacement compared with surgical aortic valve replacement in high-risk patients with Ssvere aortic stenosis: Results of the PARTNER (Placement of Aortic Transcatheter Valves) Trial (Cohort A). J Am Coll Cardiol 2012;60:2683-92.

19 Neyt M, Van Brabandt H, Devriese S, Van De Sande S. A cost-utility analysis of transcatheter aortic valve implantation in Belgium: focusing on a well-defined and identifiable population. BMJ Open 2012;2(3).

20 Doble B, Blackhouse G, Goeree R, et al. Cost-effectiveness of the Edwards SAPIEN transcatheter heart valve compared with standard management and surgical aortic valve replacement in patients with severe symptomatic aortic stenosis: A Canadian perspective. J Thorac Cardiovasc Surg 2012

21 Dimarakis I, Rehman SM, Grant SW, et al. Conventional aortic valve replacement for high-risk aortic stenosis patients not suitable for trans-catheter aortic valve implantation: feasibility and outcome. Eur J Cardiothorac Surg 2011:40:743-8.

22 Reynolds MR, Magnuson EA, Wang K, et al. Cost-Effectiveness of transcatheter aortic valve replacement compared with standard care among inoperable patients with severe aortic stenosis: results From the Placement of Aortic Transcathete Valves (PARTNER) Trial (Cohort B). Circulation 2012;125:1102-9.

23 Buellesfeld L, Gerckens U, Schuler G, et al. 2-year follow-up of patients undergoing transcatheter aortic valve implantation using a self-expanding valve prosthesis. J Am Coll Cardiol 2011:57:1650-7.

24 Tamburino C, Capodanno D, Ramondo A, et al. Incidence and predictors of early and late mortality after transcatheter aortic valve implantation in 663 patients with severe aortic stenosis. Circulation 2011;123:299-308.

25 Zahn R, Gerckens U, Grube E, et al. Transcatheter aortic valve implantation: first results from a multi-centre real-world registry. Eur Heart J 2011;32:198-204.

26 Luengo-Fernandez R, Gray AM, Rothwell PM. Costs of stroke using patient-level data: a critical review of the literature. Stroke 2009:40:e18-23.

27 Brennan A, Kharroubi S, O'Hagan A, et al. Calculating partial expected value of perfect information via Monte Carlo sampling algorithms. Med Decis Making 2007;27:448-70

28 Curtis L. Unit Costs of Health and Social Care. Personal Social Services Research Unit. 2011. http://www.pssru.ac.uk/archive/pdf/uc/uc2011/uc2011.pdf (accessed 14 Feb 2013)

29 Britain. BMAaRPSoG. British National Formulary. BMA 2011;61.

30 Moat NE, Ludman $\mathrm{P}$, de Belder MA, et al. Long-term outcomes after transcatheter aortic valve implantation in high-risk patients with severe aortic stenosis: the U.K. TAVI (United Kingdom Transcatheter Aortic Valve Implantation) Registry. J Am Coll Cardiol 2011:58:2130-8.

31 Solomon NA, Glick HA, Russo CJ, et al. Patient preferences for stroke outcomes Stroke 1994:25:1721-5.

32 Morgan SL, McEwan P, Morrissey M, et al. Characterization and comparison of health-related utility in people with diabetes with various single and multiple vascular complications. Diabet Med 2006;23:1100-5.

33 Lee AJ, Morgan CL, Conway $\mathrm{P}$, et al. Characterisation and comparison of healthrelated quality of life for patients with renal failure. Curr Med ResOpin 2005:21:1777-83. 